\title{
Associazione mafiosa e Roma Capitale
}

\author{
Antonio Tebano ${ }^{\circ}$
}

\section{Riassunto}

L'Autore rende un contributo volto a sottolineare la peculiarità dell'associazione mafiosa emersa a Roma a seguito della fusione dei gruppi Carminati e Buzzi.

Intreccio di forze criminali con le istituzioni: Comune e sue partecipate.

Condizionamento dell'attività amministrativa; modalità di pressione e risultati conseguiti (profitti illeciti).

\section{Résumé}

Dans son article, l'auteur met en évidence la particularité de l'association mafieuse qui s'est développée à Rome après la fusion des groupes Carminati et Buzzi.

Suite à cette situation, l'auteur décrit l'entrelacement des forces criminelles et des institutions : la municipalité et les sociétés en participation.

Une influence sur les tâches administratives ; moyens de pression et ses résultats (bénéfices illicites).

\section{Abstract}

The author describes the characteristics of the mafia organisation which was discovered in Rome after the unification of Carminati and Buzzi groups.

As a result of the above mentioned situation the author talks about the cooperation between criminal forces and various institutions such as the municipality and its associated companies.

Management of administrative activities; pressure methods and achieved results (illegal profits).

Key words: Mafia organization; Rome; illegal profits; pressure methods; municipality associated companies.

\footnotetext{
- Avvocato, ha iniziato la libera professione nel 1954. E' iscritto all'Albo Speciale degli Avvocati Cassazionisti. Componente del Consiglio dell' Ordine degli Avvocati di Bologna dal 1972 al 1995 e membro, in più occasioni, della Commissione per gli esami da avvocato. Insignito del riconoscimento "Toga d'oro" nell'anno 2006.
} 
Queste note traggono spunto dalla sentenza n. $24532 \mathrm{VI}^{\circ}$ Sezione penale Cassazione, emessa il 10/4/2015; la decisione, relativa alla vicenda nota "Roma Capitale", respinge i ricorsi proposti da alcune difese e conferma l'ordinanza camerale del Tribunale del Riesame di Roma del 23/12/2014, a sua volta confermativa di quella GIP Roma 28/11/2014, genetica delle misure cautelari.

Il tema decidendum, sollevato dai ricorrenti (con motivo principale comune), si incentra sulla ricorrenza nella specie, avuto riguardo alle imputazioni mosse, dell'associazione di tipo mafioso (416 bis c.p.).

La Corte, dopo un'approfondita analisi critica dei motivi sia in fatto che in diritto, perviene alla conclusione che sussiste nella fattispecie l'aggravante “dell'avere agito al fine di agevolare l'associazione criminale diretta da Carminati'.

Le motivazioni, a supporto, si fondano sui risultati del compendio indiziario delineati nell'ordinanza del 28/11/2014, ritenuti corretti e logici, convergenti a fornire la prova dell'alleanza stretta fra Carminati e Buzzi.

Le emergenze, costituite da intercettazioni telefoniche ed ambientali, documentazione contabile, tra cui il "libro nero" contenente annotazioni di pagamenti in nero e/o tangenti, comprovano - in modo univoco - la fusione fra $\mathrm{i}$ due gruppi.

Carminati è un notissimo esponente della malavita romana, legato in passato alla banda della Magliana, già militante nel nucleo armate rivoluzionarie (NAR), aderente all'estrema destra eversiva, con numerosi precedenti penali, anche per omicidio e ristretto in carcere per periodi di lunga durata.

Ha rapporti con esponenti politici di rilievo della sua area, con amministratori di primo piano del Comune e delle società partecipate (EUR s.p.a. - AMA s.p.a.) e con alti funzionari dello Stato.

Buzzi è altrettanto noto come figura apicale nel mondo cooperativo; gestisce e guida un numero notevole di cooperative che operano per il Comune e per le due società sopra citate; anche lui con precedenti penali ed esperienze carcerarie.

Le cooperative presenti sul territorio di Roma da circa 30 anni sono aggiudicatarie di importanti appalti pubblici in settori rilevanti (pulizie industriali, raccolta e smaltimento dei rifiuti, manutenzione del verde, accoglienza profughi ed immigrati in Italia).

Carminati e Buzzi per storie personali, provenienze politiche, appartengono a due mondi diversi "nero il primo, rosso il secondo"; due mondi che sul piano ideologico sarebbero agli antipodi, ma, grazie all'intuito criminale dei due, riescono a compenetrarsi e a fondersi in una "cosa nuova", che rappresenta il "salto di qualità" dell'organizzazione Carminati.

Il collante che unisce le due realtà (la prima braccio armato, la seconda braccio operativo) è la penetrazione - sempre più massiccia e visibile (influenza intimidatoria) nei gangli vitali dell'amministrazione capitolina e delle "partecipate"; sul peso economico e politico di queste è sufficiente ricordare che l'azienda dei trasporti romani (AMA) conta mediamente tra i 12/13 mila dipendenti. 
L'avvenuta fusione si coglie nelle seguenti circostanze :

1) partecipazione costante del Carminati, con ruolo decisionale, alle riunioni che si tengono presso la Società ERICHES;

2) utilizzo di utenze e dispositivi elettronici sofisticati, forniti dal Carminati, allo scopo di eludere eventuali attività investigative ;

3) ripartizione dei profitti (al Carminati spetta il 50\% pari ad un ammontare di un milione di euro);

4) scambio continuo di informative riguardanti le scelte strategiche del gruppo e le modalità di risoluzione di problematiche insorte in esecuzione dei lavori;

5) rapporto di estrema fiducia fra $i$ due; sintomatica la circostanza dell'affidamento in custodia da Carminati a Buzzi (nel timore di un arresto), della somma di 500.000,00 Euro; importo che poi viene impiegato per la gestione dei campi nomadi di Castel Romano, attività anche questa gestita dalla "29 giugno".

Per effetto della fattiva partecipazione del Carminati alla gestione delle Cooperative e alla conseguente suddivisione degli utili, gli viene attribuita la qualifica di "amministratore di fatto", contrariamente a quanto figura formalmente - socio lavoratore nella cooperativa 29 giugno .

Proprio con riguardo alla predetta cooperativa, "ironia della sorte" emerge che presso la stessa lavorano 500 ex detenuti, tra cui ex terroristi e condannati per reati di vario genere, tutte persone ben conosciute dal Carminati nell' ambiente carcerario nel quale si muove perfettamente a suo agio, stimato e rispettato.

Sta di fatto che la nuova organizzazione opera in un primo momento nei settori storici della criminalità, estorsione, usura, recupero crediti (anche con atti di violenza). E' il caso di alcuni imprenditori privati in difficoltà (Ietto, Guarnera, Guaglianone, operanti il primo nel settore della ristorazione, il secondo in quello dell'edilizia, il terzo in quello del movimento terra) che chiedono aiuto e protezione al Carminati, li ottengono eseguendo lavori per le cooperative in subappalto.

Da quel momento i predetti imprenditori diventano "meri esecutori" al soldo del Carminati, solo per il quale devono lavorare.

L'occhio vigile ed attento di Carminati è rivolto in modo particolare alle vicende politicoamministrative della Capitale e si allerta nel momento in cui, alla guida del Comune nel 2008, si insedia la nuova compagine politica (destra), risultata vincente alle elezioni.

In questo contesto il Carminati vede emergere in posizioni di primo piano sia in Comune che nelle partecipate suoi vecchi e fidati amici, della sua stessa fede politica (Pucci, Gramazio, Panzironi); a questo punto si spalancano le porte del Campidoglio per Carminati, che non trova alcun ostacolo e/o concorrenza, forte anche della piena disponibilità del fronte cooperativo.

Gli affari vanno a gonfie vele, i profitti aumentano in modo esponenziale; il fatturato di 26 milioni del 2010, passa a quello di 50 milioni del 2013, quasi raddoppiandosi nel giro di un triennio.

Va da sé che questo progressivo incremento di commesse, e conseguimento di utili, può 
realizzarsi solo con la collusione di "funzionari pubblici corrotti", che sovraintendono ai settori nei quali operano le cooperative.

Luca Odevaine, in effetti originariamente Odevane (si aggiunge una "i" e si cambia persona), componente del Tavolo di Coordinamento sull'immigrazione istituito presso il Ministero dell'Interno, settore in cui opera la 29 giugno, percepisce una retribuzione a suo nome mensile di Euro 5.000, proveniente dalle casse delle Cooperative, mentre ne riceve un'altra di 1.500 Euro, sempre dalla stessa provenienza, destinata a Schina, che ha la funzione di intermediario fra Odevaine e Buzzi.

Da questi dati documentati, è evidente che si è in presenza di una perversa triangolazione, che vede impegnati Carminati, Buzzi e Odevaine; il che realizza appieno il "programma Carminati". Questi, nella conversazione dell'11 gennaio 2013, con Brugia e Guarnera enuncia il suo "manifesto" con la rappresentazione metaforica di quattro mondi, quello di mezzo in cui "tutti si incontrano" compresa l'associazione, con la funzione di garante di illecite forme di "osmosi" fra il mondo di sopra (imprenditori, politica, istituzioni) e il mondo di sotto (la malavita di strada), capace di conseguire "risultati" che interessano al sovra mondo (quello di pochi onnipotenti che tutto possono fare).

A tale riguardo il pensiero va ai "cerchi infernali danteschi”.

In attuazione del "programma" la forza dell'associazione diventa sempre più rilevante, condizionando da un lato l'azione della Pubblica Amministrazione, in violazione dei principi dell'imparzialità e trasparenza, e quella dell'imprenditoria privata in violazione della libertà di iniziativa economica e di concorrenza; principi quelli sopra enunciati, cardine della nostra costituzione.

In questo quadro e con le peculiarità rappresentate dal caso in esame, la Corte coglie i caratteri propri della "mafiosità".

A commento, lo scrivente (da un osservatorio privilegiato di oltre cinquant'anni di esercizio professionale - di cui gli ultimi trenta da impegno in processi per vicende altrettanto rilevanti) condivide il principio fissato nella sentenza in uno alle "novità" per la nozione di associazione di stampo mafioso (416 bis).

Rafforzato ancora di più nel convincimento che la mafia nelle sue varie articolazioni - Cosa Nostra, n'drangheta, Camorra, Sacra Corone Unite - costituisce un flagello nazionale, il "morbo italico" che, a parte gli effetti negativi sulla Pubblica Amministrazione, sull'economia sana, ha prodotto e continua a produrre discredito all'estero verso il nostro Paese.

\section{Riferimenti bibliografici.}

- De Francesco G., "Gli artt.416, 416bis, 416ter, 417 e 418 c.p.”, Corso P., Insolera G., Stortoni L. (a cura di), Mafia e criminalità organizzata, UTET, Torino, 1995, vol. I, pp. 5 e ss.

- De Liguori L., "La struttura normativa dell'associazione di tipo mafioso", in Cassazione penale, 1988, pp. 1611-1614.

- Insolera G., Diritto penale e criminalità organizzata, il Mulino, Bologna, 1996.

- Neppi Modona G, "Il reato di associazione mafiosa", in Democrazia e diritto, 1983. 